Supporting Information for

\title{
Molecular Level Structural Characterization of Self-Assembled Monolayers of Functionalized Bidentate Aromatic Thiols
}

\author{
Umit Celik, ${ }^{\#}$ Han Ju Lee, ${ }^{2}$ Terell Keel, ${ }^{1}$ Logan A. Swartz, ${ }^{3}$ Marshall Van Zijll, ${ }^{1}$ Cody J. \\ Chalker, ${ }^{1}$ T. Randall Lee, ${ }^{2, *}$ Gang-yu Liu ${ }^{1,3^{*}}$ \\ 1Department of Chemistry, University of California, Davis, California, 95616, United States \\ 2Department of Chemistry and the Texas Center for Superconductivity, University of Houston, 4800 Calhoun Road, \\ Houston, Texas 77204, United States \\ 3Biophysics Graduate Group, University of California, Davis, California, 95616, United States
}

\section{Table of Contents}

1. Materials for Imaging and Structural Characterization...........................

2. Synthesis of (5-(Octyloxy)-1,3-phenylene)dimethanethiol $\ldots \ldots \ldots \ldots \ldots \ldots \ldots \ldots . . . .6$

3. Preparation of SAMs on Gold Thin Films................................. -6

4. STM Imaging and Structural Characterization ...............................

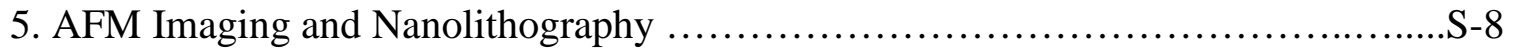

6. Schematic Diagram Generated Using Chem3D.............................

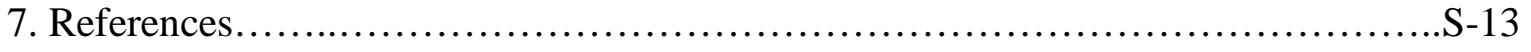

\section{AUTHOR INFORMATION}

*E-mails: gyliu@ucdavis.edu, trlee@uh.edu 


\section{Materials for Imaging and Structural Characterization}

Octadecane-1-thiol (abbreviated as $\mathrm{C}_{18} \mathrm{SH}$ ) (98\%), and decane-1-thiol (abbreviated as $\mathrm{C}_{10} \mathrm{SH}$ ) (98\%), were obtained from Sigma Aldrich (St. Louis, MO, U.S.A) and used as received. The clear ruby muscovite mica substrate was purchased from Mica New York Corp (NY, U.S.A.). Ethanol (200 proof), decalin (a mixture of cis and trans, 98\%), hexane ( $\geq 99.9 \%$ ), $\mathrm{KOH}$, tetrahydrofuran (THF, purity $\geq 99.9 \%$ ) were all purchased from Sigma Aldrich. Deionized water ( $\geq 18.2 \mathrm{M} \Omega$ ) was purified by a Milli-Q system (Q-GARD 2, Millipore Sigma, Billerica, MA, U.S.A.). The STM tip materials were tungsten wires (purity 99.95\%) purchased from California Fine Wire Company (Grover Beach, CA, U.S.A.).

\section{Synthesis of (5-(Octyloxy)-1,3-phenylene)dimethanethiol}

Materials for synthesis. 3.5-Bis(hydroxymethyl)phenol was prepared according to procedures available in the literature. ${ }^{1}$ Potassium carbonate $\left(\mathrm{K}_{2} \mathrm{CO}_{3}\right)$, methanesulfonyl chloride (MsCl), triethylamine $\left(\mathrm{Et}_{3} \mathrm{~N}\right)$, potassium thioacetate, lithium aluminum hydride $\left(\mathrm{LiAlH}_{4}\right)$, and 1iodooctane were purchased from Sigma-Aldrich and used as received. The solvents tetrahydrofuran (THF) and dichloromethane $\left(\mathrm{CH}_{2} \mathrm{Cl}_{2}\right)$ were purchased from Sigma-Aldrich. Hexanes, diethyl ether, acetonitrile $\left(\mathrm{CH}_{3} \mathrm{CN}\right)$, and methanol $(\mathrm{MeOH})$ were purchased from Mallinckrodt Chemicals. A saturated $\mathrm{NaCl}$ aqueous solution was used as the brine and was prepared by dissolving crystalline $\mathrm{NaCl}$ (Mallinckrodt Chemicals) into pure water until saturation, i.e., with some $\mathrm{NaCl}$ crystals remained on the bottom of the bottle. Anhydrous ethanol (EtOH) was purchased from Decon Labs, Inc. Distilling over calcium hydride gave dry tetrahydrofuran and dichloromethane. Silica gel for column chromatography was obtained from Sorbent Technologies. 
Procedures. (5-(Octyloxy)-1,3-phenylene)dimethanethiol (OPDT) was prepared using the steps outlined in Scheme S1. The experimental details are provided in the subsequent paragraphs.

Scheme S1. Synthesis of (5-(Octyloxy)-1,3-phenylene)dimethanethiol (OPDT)
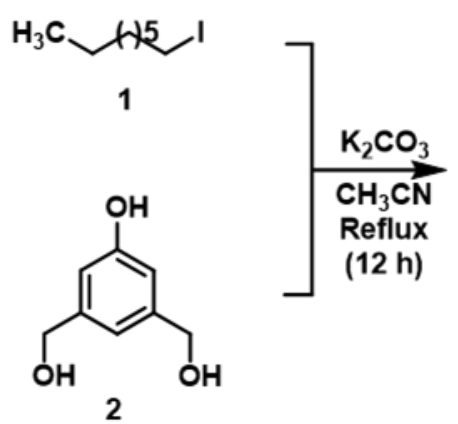

$(12 \mathrm{~h})$

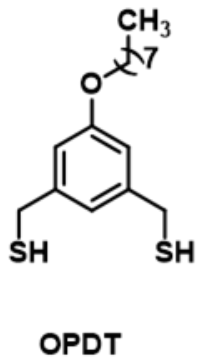

OPDT

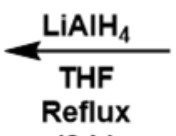

$(2 \mathrm{~h})$

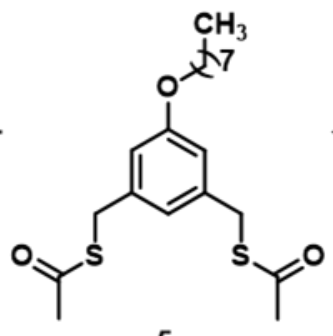

5
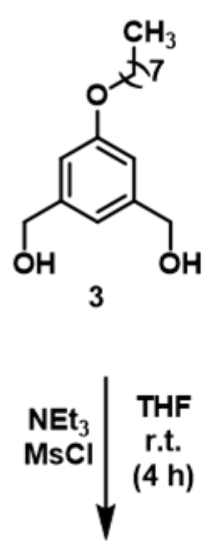

3

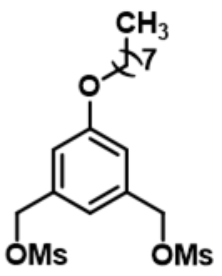

4

(5-(Octyloxy)-1,3-phenylene)dimethanol (3). A mixture of $\mathrm{K}_{2} \mathrm{CO}_{3}$ (3.76 g, $20.0 \mathrm{mmol}$ ), 1iodooctane (1.93 g, $8.04 \mathrm{mmol})$, and 3.5-bis(hydroxyl-methyl)phenol (1.40 g, $9.09 \mathrm{mmol})$ in acetonitrile $(250 \mathrm{~mL})$ was refluxed for $12 \mathrm{~h}$. The resulting mixture was condensed by rotary evaporation followed by the addition of diethyl ether $(200 \mathrm{~mL})$ and then acidification with $4.0 \mathrm{M}$ $\mathrm{HCl}$ solution to afford a solution $\mathrm{pH}$ of $\sim 3$. The organic layer was washed with water $(3 \times 100$ $\mathrm{mL}$ ), dried over $\mathrm{Na}_{2} \mathrm{SO}_{4}$, and concentrated in vacuo to produce $1.90 \mathrm{~g}$ (7.14 mmol) of a white solid (89\% yield). ${ }^{1} \mathrm{H}$ NMR (500 MHz, $\left.\mathrm{CDCl}_{3}\right): \delta 6.24$ (s, 1H), 6.84 (s, 2H), 4.66 (s, 4H), $3.96(\mathrm{t}, J=$ $6.4 \mathrm{~Hz}, 2 \mathrm{H}), 1.76$ (m, 2H), 1.25-1.43 (m, 26H), 0.87 (t, $J=6.8 \mathrm{~Hz}, 3 \mathrm{H})$. 
(5-(Octyloxy)-1,3-phenylene)bis(methylene) dimethanesulfonate (4). A 1.90 g (7.14 mmol) aliquot of (5-(octyloxy)-1,3-phenylene)dimethanol was introduced into a 500-mL round-bottomed flask containing $250 \mathrm{~mL}$ of THF, and then triethylamine ( $3.2 \mathrm{~mL}, 30 \mathrm{mmol})$ was added slowly to the mixture, which was stirred at rt for 30 min. The reaction flask was placed in an ice bath, and methanesulfonyl chloride ( $2.1 \mathrm{~mL}, 30 \mathrm{mmol})$ was transferred slowly to the solution while stirring. The ice bath was then removed, and the mixture was stirred for $4 \mathrm{~h}$ at rt. After completion of the reaction, excess methanesulfonyl chloride was destroyed by adding $100 \mathrm{~mL}$ of water followed by acidification with $4.0 \mathrm{M} \mathrm{HCl}$ solution to afford a solution $\mathrm{pH}$ of $\sim 3$. The mixture was extracted with diethyl ether $(3 \times 100 \mathrm{~mL})$. The combined organic layers were washed with brine $(100 \mathrm{~mL})$ and water $(3 \times 100 \mathrm{~mL})$. The organic layer was dried over $\mathrm{Na}_{2} \mathrm{SO}_{4}$, filtered, and concentrated in vacuo to produce (5-(octyloxy)-1,3-phenylene)bis(methylene) dimethanesulfonate (2.70 g, 5.92 mmol), which was used in the next step without further purification (83\% yield). ${ }^{1} \mathrm{H}$ NMR (500 MHz, $\mathrm{CDCl}_{3}$ ): $\delta 7.00$ (s, 1H), 6.95 (s, 2H), 5.19 (s, 4H), 3.96 (t, J = $\left.6.4 \mathrm{~Hz}, 2 \mathrm{H}\right), 2.97$ (s, 3H), 1.77 (m, 2H), 1.28-1.44 (m, 10H), 0.88 (t, $J=7.3 \mathrm{~Hz}, 3 \mathrm{H})$.

S,S'-(5-(Octyloxy)-1,3-phenylene)bis(methylene) diethanethioate (5). A mixture of potassium thioacetate (1.71 g, $15.0 \mathrm{mmol})$ and (5-(octyloxy)-1,3-phenylene)bis(methylene) dimethanesulfonate ( $2.70 \mathrm{~g}, 5.92 \mathrm{mmol})$ in ethanol $(300 \mathrm{~mL})$ was refluxed for $12 \mathrm{~h}$ under nitrogen. After cooling, the precipitate was filtered off, rinsed with ethanol, and the combined organic phases were evaporated to dryness. The residue was taken up in a blend of diethyl ether $(100 \mathrm{~mL})$ and water $(200 \mathrm{~mL})$. The mixture was extracted with diethyl ether $(2 \times 100 \mathrm{~mL})$. The combined organic layers were washed with brine $(3 \times 100 \mathrm{~mL})$, dried over anhydrous $\mathrm{Na}_{2} \mathrm{SO}_{4}$, filtered, and concentrated by rotary evaporation, and then dried in vacuo to give $1.25 \mathrm{~g}$ (3.28 mmol) of S,S'-(5(octyloxy)-1,3-phenylene)bis(methylene) diethanethioate as a yellow solid (55\% yield). ${ }^{1} \mathrm{H}$ NMR 
(500 MHz, $\mathrm{CDCl}_{3}$ ): $\delta 6.75$ (s, 1H), 6.69 (s, 2H), 4.03 (s, 4H), 3.89 (t, J = 6.4 Hz, 2H), 2.33 (s, 6H), 1.77 (m, 2H), 1.25-1.44 (m, 10H), 0.87 (t, $J=7.3 \mathrm{~Hz}, 3 \mathrm{H})$.

(5-(Octyloxy)-1,3-phenylene)dimethanethiol (OPDT). To a suspension of $\mathrm{LiAlH}_{4}(0.380$ g, 10.0 mmol) in THF (300 mL) was added a solution of $S, S^{\prime}$-(5-(octyloxy)-1,3-phenylene)bis(methylene) diethanethioate $(1.25 \mathrm{~g}, 2.28 \mathrm{mmol})$ in THF $(50 \mathrm{~mL})$ dropwise at $0{ }^{\circ} \mathrm{C}$. The ice bath was then removed, and the mixture was refluxed for $2 \mathrm{~h}$ under nitrogen. Subsequently, the reaction was quenched with degassed ethanol $(25 \mathrm{~mL})$. The mixture was acidified to $\mathrm{pH} \sim 1$ by careful addition of 2.0 M HCl solution and then extracted with diethyl ether $(3 \times 100 \mathrm{~mL})$. The combined organic layers were washed with brine $(1 \times 100 \mathrm{~mL})$, dried over anhydrous $\mathrm{Na}_{2} \mathrm{SO}_{4}$, filtered, and concentrated by rotary evaporation. The crude product was purified by column chromatography (hexanes:ethyl acetate $=9.8: 0.2)$ to give $0.605 \mathrm{~g}(2.18 \mathrm{mmol})$ of OPDT as a colorless oil $(66 \%$ yield). ${ }^{1} \mathrm{H}$ NMR (500 MHz, $\left.\mathrm{CDCl}_{3}\right): \delta 6.84$ (s, 1H), 6.75 (s, 2H), 3.94 (t, $\left.J=6.3 \mathrm{~Hz}, 2 \mathrm{H}\right), 3.68$ (d, $J=8.0 \mathrm{~Hz}, 4 \mathrm{H}), 1.76(\mathrm{~m}, 4 \mathrm{H}), 1.27-1.45(\mathrm{~m}, 10 \mathrm{H}), 0.89(\mathrm{t}, J=6.3 \mathrm{~Hz}, 3 \mathrm{H}) .{ }^{13} \mathrm{C}$ NMR $(125 \mathrm{MHz}$, $\left.\mathrm{CDCl}_{3}\right): \delta$ 159.72, 143.00, 119.90, 112.96, 68.11, 31.94, 29.48, 29.38, 29.04, 26.18, 22.80, 14.27.

\section{${ }^{1} \mathrm{H}$ and ${ }^{13} \mathrm{C}$ NMR Spectra.}




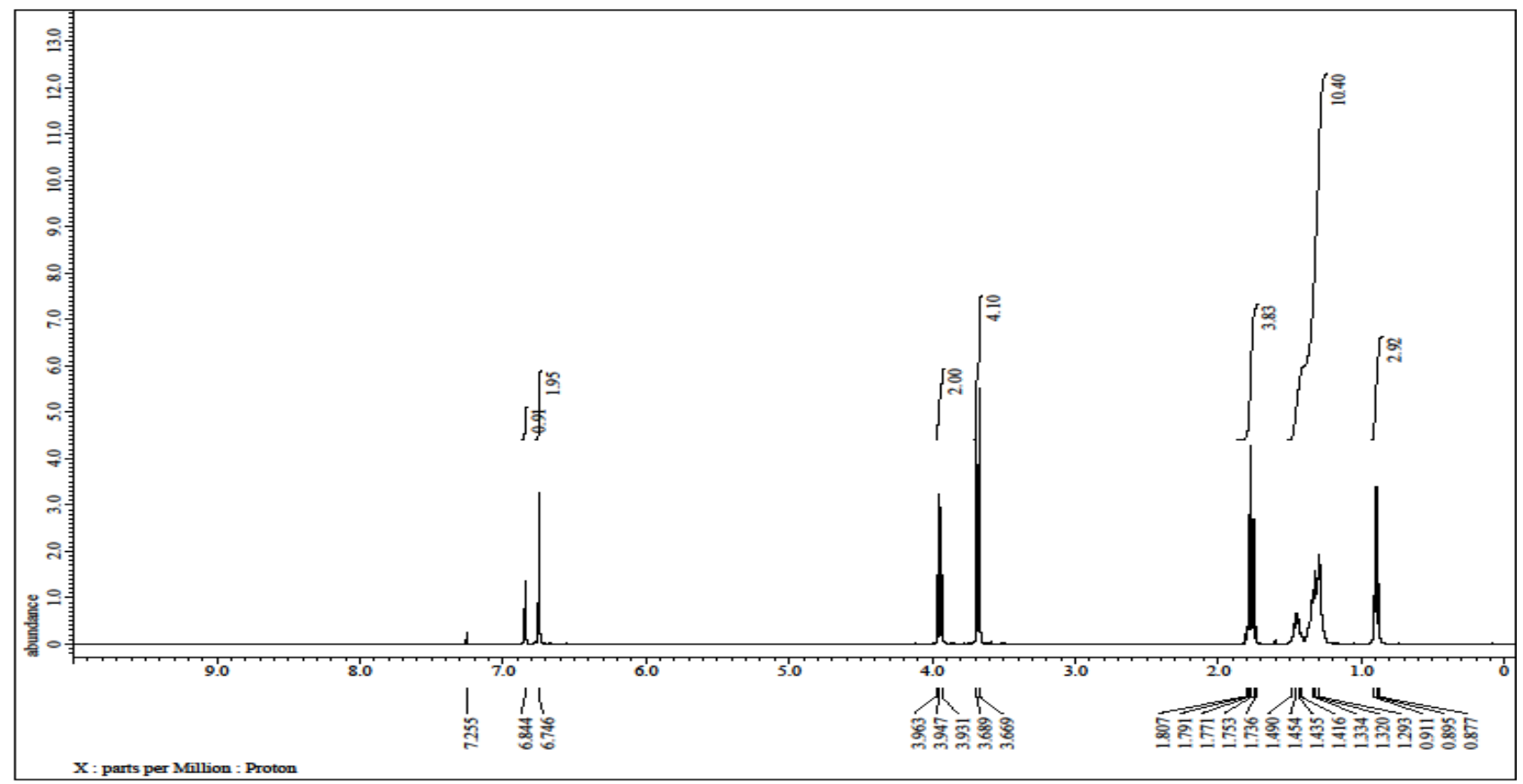

Figure S1. ${ }^{1} \mathrm{H}$ NMR spectrum of (5-(octyloxy)-1,3-phenylene)dimethanethiol (OPDT).

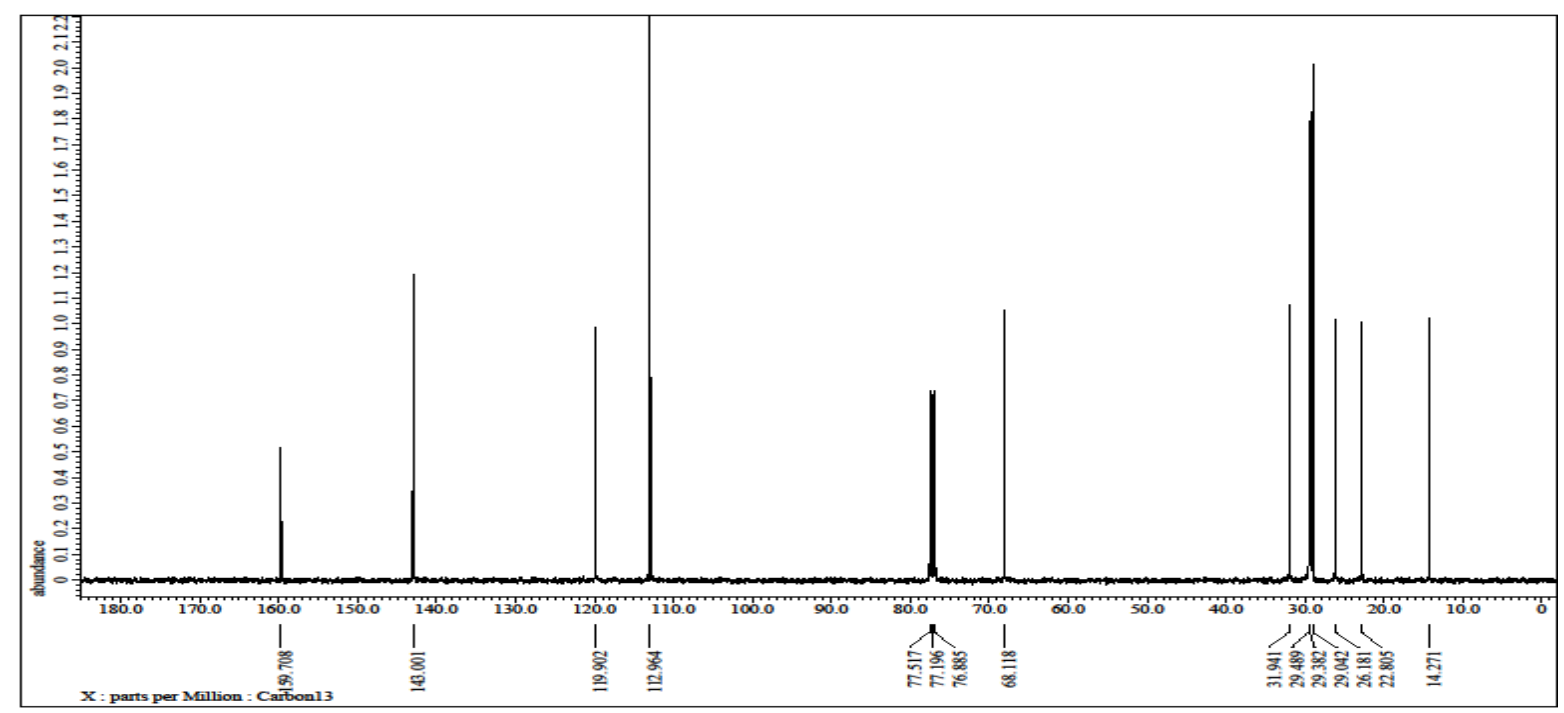

Figure S2. ${ }^{13} \mathrm{C}$ NMR spectrum of (5-(octyloxy)-1,3-phenylene)dimethanethiol (OPDT). 


\section{Preparation of SAMs on Gold Thin Films}

$\mathrm{Au}(111)$ thin films were prepared in a high vacuum evaporator (DV-502A Thermal Evaporation system, Denton Vacuum, Moorestown, NJ, U.S.A.). ${ }^{2}$ The evaporation rate was $1.9 \AA$ Ås. The mica substrate was maintained at $673 \mathrm{~K}$ during Au deposition. After evaporation, the films were annealed at $673 \mathrm{~K}$ for 30 minutes. These protocols are known to yield $\mathrm{Au}(111)$ terraces, hundreds of nanometers in lateral dimensions. ${ }^{2}$

In parallel, a $1 \mathrm{mM}$ decanethiol and a $1 \mathrm{mM}$ octadecanethiol solution in ethanol were prepared. A $1 \mathrm{mM}$ OPDT solution in THF was also prepared. ${ }^{3}$ Mild sonication was used, if necessary, to accelerate the dissolution of the adsorbate. The Au thin films taken from the evaporator were cleaned using hydrogen flaming, then immediately immersed into the designated solutions (see above) for at least 24 hours. ${ }^{2}$ Prior to imaging, the surfaces of the thiol SAMs were washed with ethanol, hexane, then ethanol again. The samples were transferred to the designated sample stages for the STM and AFM investigations upon drying. The OPDT SAMs on Au(111) were washed sequentially with ethanol, decalin, hexane, ethanol, and then dried before being transferred to sample stages for STM or AFM investigations.

\section{STM Imaging and Structural Characterization}

Upon removal from the ethanolic OPDT solution and washing following the protocols described in Section 3 above, the OPDT SAMs were immediately mounted and transferred to the UHV STM chamber. ${ }^{2,4}$ The STM tips used for this study were prepared by mechanically cutting tungsten wires, followed by electrochemical etching. ${ }^{2,4}$ A home-constructed electrochemical potentiostat was used to control the redox reaction time and current to avoid over-etching. ${ }^{2,4}$ The potentiostat monitored the redox current and automatically halted the etching process when the current dropped 
suddenly. Typical etching conditions were $2.1 \mathrm{~V}$ in a $3 \mathrm{M} \mathrm{KOH}$ solution. ${ }^{2}$ The freshly fabricated tips were then washed sequentially with Milli-Q water and then ethanol several times. The tips were then quickly transferred to the UHV STM chamber.

A variable-temperature STM (STM 300, RHK Technology, Inc., Troy, MI, U.S.A.) was employed for imaging. The STM was operated under UHV conditions with the background pressure in the UHV chamber maintained at $1 \times 10^{-10}$ mbar. A tungsten filament heater was mounted underneath the sample stage for annealing, and the final temperatures (300-398 K) were adjusted by varying the current. Temperatures were monitored using a thermocouple ( $\mathrm{K}$ type) installed directly underneath the sample to measure and monitor the annealing process. ${ }^{2,4}$

The conditions for imaging the OPDT SAMs ranged from \pm 1.2 to $\pm 1.4 \mathrm{~V}$ bias voltage and 5 to 20 pA tunneling current. All images reported in this work were acquired in a constant-current mode. All SAMs were initially imaged at room temperature as illustrated, for example, in Figure S3 for the $\mathrm{C}_{10} \mathrm{~S} / \mathrm{Au}(111) \mathrm{SAM}$, where the characteristic 2D closely packed structure is clearly visible with a periodicity consistent with the well-known $(\sqrt{3} \mathrm{x} \sqrt{ } 3) \mathrm{R} 30^{\circ}$ structure. $^{2,}{ }^{4}$ The inset shows the corresponding FFT imaging revealing the hexagonal symmetry and periodicity. For the annealing experiments reported in this work, SAMs were heated from room temperature to the desired annealing temperature at a rate of about $1 \mathrm{~K} / \mathrm{min}$. After reaching the chosen temperature and being maintained for the designated period of time, the samples were then cooled for $2-5 \mathrm{~h}$ to room temperature prior to STM imaging. 


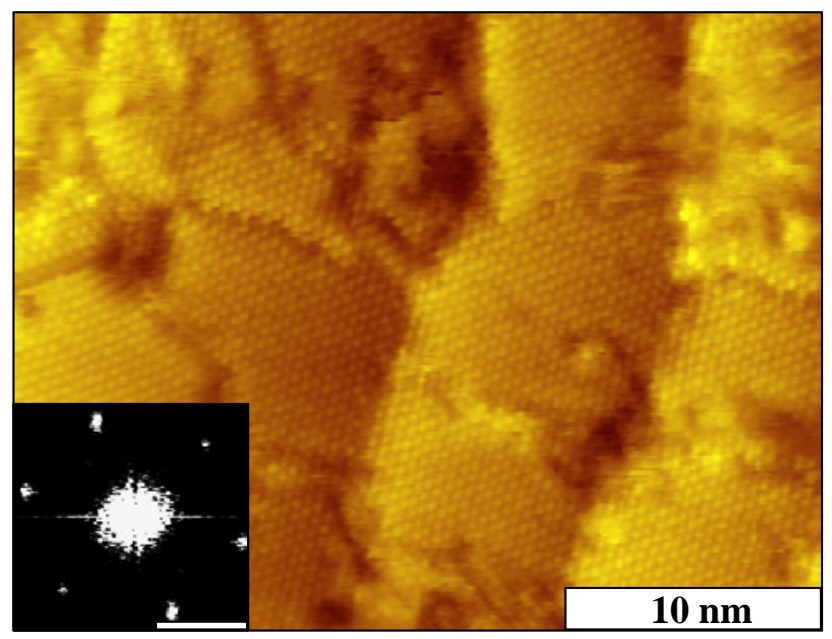

Figure S3. A $30 \mathrm{~nm}$ x $23 \mathrm{~nm}$ STM topographic image of $\mathrm{C}_{10} \mathrm{SH}$ SAM imaged under UHV, at bias voltages of $V=1 \mathrm{~V}$, and tunneling current $\mathrm{I}=20 \mathrm{pA}$. Scale bar on FFT $2 \mathrm{Gm}^{-1}$.

\section{AFM Imaging and Nanolithography}

The atomic force microscope (AFM) used was a commercial unit (MFP-3D, Oxford Instruments, Santa Barbara, CA, U.S.A.). Microfabrication cantilevers (MSLN-10, Bruker, Camarillo, California, USA) were used for all imaging and nanolithography experiments. The force constant of each cantilever was provided by the manufacturer $(0.10 \mathrm{~N} / \mathrm{m})$ and recalibrated prior to imaging by measuring its resonance frequency. ${ }^{5-6}$ The SAMs were imaged via contact mode under a load of $0.5 \mathrm{nN}$ in ambient, and $0.1 \mathrm{nN}$ in liquid media, respectively. Thicknesses of the OPDT SAMs were determined by imaging and comparing them to alkanethiol SAMs of known thickness. The alkanethiol SAMs served as internal references and were inlaid in the OPDT SAMs using nanografting. ${ }^{7-8}$

Nanografting was developed by our team and reported previously. ${ }^{7-9}$ Briefly here, an OPDT SAM was first imaged using AFM in an ethanol media containing $1 \mathrm{mM} \mathrm{C}_{18} \mathrm{SH}$. These initial AFM images revealed the OPDT SAM topography and allowed nanografting sites to be selected. Next, 
the probe was parked above the selected location and approached until a designated high load or pressure was achieved (e.g., $40 \mathrm{nN}$ ). Under this high force, the OPDT adsorbates (beneath the probe-surface contact) were displaced or shaved during the scan. The alkanethiol molecules above were attached to the newly exposed $\mathrm{Au}$ area, thus forming an area of $\mathrm{C}_{18} \mathrm{SH}$ SAM inlaid in the OPDT SAM. For this experiment, ethanol solution was used.

The results of the thin-film height measurements are discussed in the main text, and the section below details the quantification of the uncertainties pertaining to these measurements. Uncertainties in z-values from the AFM topographic images propagate to the height difference determination between the $\mathrm{C}_{18} \mathrm{SH}$ and OPDT SAMs following simple calculations in eqs (1) and (2) below,

$$
\begin{aligned}
& \Delta \mathrm{h}=\mathrm{h} 1-\mathrm{h} 2 \\
& \delta_{\Delta \mathrm{h}}=\sqrt{\delta_{\mathrm{h} 1}^{2}+\delta_{\mathrm{h} 2}^{2}}
\end{aligned}
$$

where, $\mathrm{h} 1$ and $\mathrm{h} 2$ are the apparent height of the $\mathrm{C}_{18} \mathrm{SH}$ and OPDT SAMs measured directly from the AFM topographic images, respectively, and $\Delta \mathrm{h}$ denotes the difference in layer thickness between the two SAMs. Eq. (2) is the error propagation following eq. (1), where the $\delta_{\mathrm{h} 1}, \delta_{\mathrm{h} 2}$, and $\delta_{\Delta \mathrm{h}}$ represent the uncertainties in $\mathrm{h} 1, \mathrm{~h} 2$, and $\Delta \mathrm{h}$, respectively.

In the case of the $\mathrm{C}_{18} \mathrm{SH}$ SAM nanografted into an OPDT SAM, the values of $p$ and $q$ are 1.08 and $0.025 \mathrm{~nm}$, respectively, determined from the AFM image shown in Figure 6 in the main text. The same images also yielded $\delta_{\mathrm{p}}$ and $\delta_{\mathrm{q}}$ values to be 0.076 and $0.058 \mathrm{~nm}$, respectively. Therefore, $\Delta \mathrm{h}$ and $\delta_{\mathrm{x}}$ can be calculated using eqs. (1) and (2): 1.055 and $0.096 \mathrm{~nm}$, respectively. The structure of $n$-alkanethiol SAMs is well known: 2D closely packed chains with a periodicity of $0.499 \mathrm{~nm}$ and chains tilting of $30.5^{\circ}$ from surface normal. ${ }^{10}$ Therefore, the $\mathrm{C}_{18} \mathrm{SH}$ SAM thickness equals alkane 
chain-length $x \cos \left(\right.$ tilt angle) + S-Au distance, as illustrated in Figure S4 $\left[2.406 \mathrm{x} \cos \left(30.5^{\circ}\right)+\right.$ $0.220=2.293 \mathrm{~nm}]$. Since the heights of the $\mathrm{C}_{18} \mathrm{SH}$ and OPDT SAMs differ by $1.055 \mathrm{~nm}$, the OPDT thickness equals $1.238 \pm 0.096 \mathrm{~nm}$, following eqs. (1) and (2).

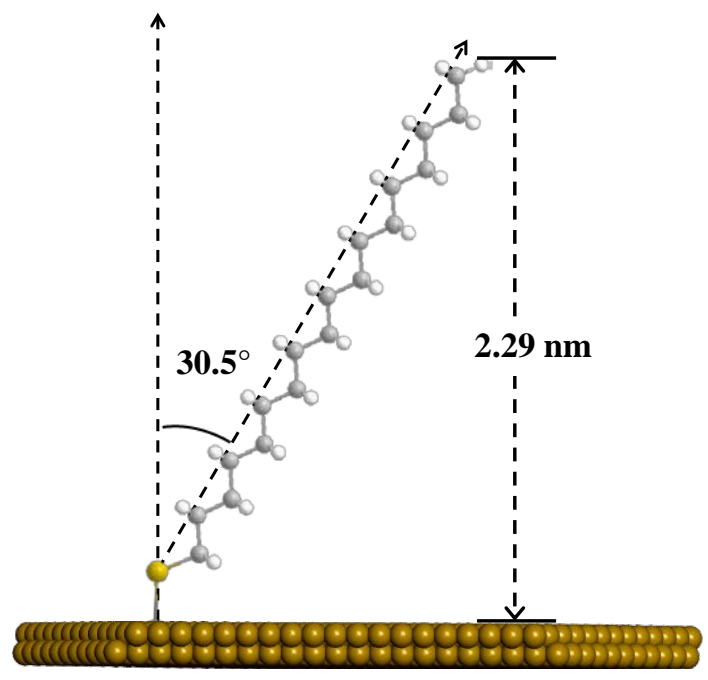

Figure S4. An octadecane-1-thiol molecule on $\mathrm{Au}(111)$, constructed from the known SAM structure. ${ }^{10}$ The monolayer thickness is illustrated in correlation with the molecular conformation. The molecular model was generated using Chem3D.

The measured OPDT thickness, $1.238 \mathrm{~nm}$, is smaller than the length of an OPDT molecule plus the sulfur gold bond $(1.83 \mathrm{~nm})$. Based on the SAM thickness measurements, two possible OPDT SAM structures follow: (a) the difference is purely due to the tilt of OPDT chains with respect to the surface normal; (b) the density of OPDT SAMs is less than a 2D closely packed structure; thus, these SAMs deform more than alkanethiol SAMs under an AFM probe under contact imaging mode. Assuming the first possibility, the tilt angle can be calculated using eqs. (3) and (4), where the angle $\theta$ represents the tilt angle of the chain, and $\delta \theta$ represents its uncertainty. In the case of OPDT SAM, $\theta=\arccos \left(\frac{1.018}{1.61}\right)=50.78^{\circ}$, illustrated in Figure S5. The uncertainty, $\delta \theta$, can be 
calculated by using error propagation, as shown in eq. (4), $\delta \theta=4.4^{\circ}$. "A" is defined as the SAM thickness minus the sulfur gold bond length divided by the molecular length $\left(\mathrm{A}=\left(\frac{1.018}{1.61}\right)\right)$.

$$
\begin{gathered}
\theta=\arccos \left(\frac{\text { SAM thickness -S-Au bond, } h}{\text { Molecular length, } \mathrm{l}}\right) \\
\delta \theta=\mathrm{A}\left(\frac{180}{\pi}\right)\left(\frac{-1}{\sqrt{1-A^{2}}}\right) \sqrt{\left(\frac{\delta \mathrm{x}}{\mathrm{x}}\right)^{2}+\left(\frac{\delta \mathrm{y}}{\mathrm{y}}\right)^{2}}
\end{gathered}
$$

As discussed in the main text, model (a) shown in Figure S5 was ultimately ruled out based on additional evidence collected in our molecular resolution STM imaging. Model (b), which consists of mixed standing up and lying-down OPDT molecules on the surface, more accurately reflects the combined STM and AFM data. Therefore, the deduction process of the model (a) was only included in the SI to report the deduction and data analysis process.

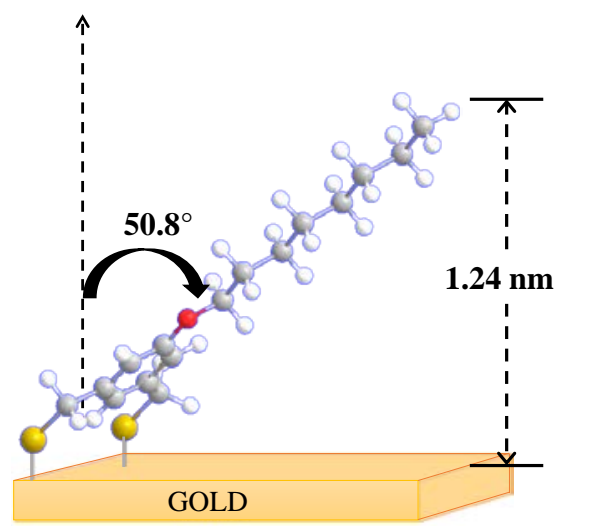

Figure S5. A possible model of OPDT SAMs on gold, where the tilt angle was calculated from AFM imaging and assumption (a).

\section{Schematic Diagram Generated Using Chem3D}

All schematic diagrams of mono- and di-thiol molecules on surfaces were generated using Chem3D 19.0 (Perkin-Elmer). It is known that sulfur atoms attach to triple hollow sites of Au(111) 
with $0.50 \mathrm{~nm}$ separation, ${ }^{4,11-12}$ which was introduced in our initial building of the OPDT in Chem3D, and held constant in subsequent energy minimization. The conformation of the adsorbate molecules was generation by energy minimization using molecular mechanics 2 (MM2) force field developed by the Allinger team. ${ }^{13}$

\section{References}

1. Lee, H. J.; Jamison, A. C.; Yuan, Y.; Li, C.-H.; Rittikulsittichai, S.; Rusakova, I.; Lee, T. R., Robust Carboxylic Acid-Terminated Organic Thin Films and Nanoparticle Protectants Generated from Bidentate Alkanethiols. Langmuir 2013, 29 (33), 10432-10439.

2. Qian, Y.; Yang, G.; Yu, J.; Jung, T. A.; Liu, G.-y., Structures of Annealed Decanethiol SelfAssembled Monolayers on $\mathrm{Au}(111)$ : an Ultrahigh Vacuum Scanning Tunneling Microscopy Study. Langmuir 2003, 19 (15), 6056-6065.

3. Hutter, J. L., Comment on Tilt of Atomic Force Microscope Cantilevers: Effect on Spring Constant and Adhesion Measurements. Langmuir 2005, 21 (6), 2630-2632.

4. Yang, G.; Liu, G.-y., New Insights for Self-Assembled Monolayers of Organothiols on Au(111) Revealed by Scanning Tunneling Microscopy. The Journal of Physical Chemistry B 2003, 107 (34), 8746-8759.

5. Hutter, J. L.; Bechhoefer, J., Calibration of atomic-force microscope tips. Review of Scientific Instruments 1993, 64 (7), 1868-1873.

6. Liu, M.; Amro, N. A.; Liu, G.-y., Nanografting for Surface Physical Chemistry. Annual Review of Physical Chemistry 2008, 59 (1), 367-386.

7. Xu, S.; Liu, G.-y., Nanometer-Scale Fabrication by Simultaneous Nanoshaving and Molecular Self-Assembly. Langmuir 1997, 13 (2), 127-129. 
8. Liu, G.-Y.; Xu, S.; Qian, Y., Nanofabrication of Self-Assembled Monolayers Using Scanning Probe Lithography. Accounts of Chemical Research 2000, 33 (7), 457-466.

9. Rittikulsittichai, S.; Park, C. S.; Marquez, M. D.; Jamison, A. C.; Frank, T.; Wu, C.-H.; Wu, J. I.; Lee, T. R., Inhibiting Reductive Elimination as an Intramolecular Disulfide Dramatically Enhances the Thermal Stability of SAMs on Gold Derived from Bidentate Adsorbents. Langmuir 2018, 34 (23), 6645-6652.

10. Fenter, P.; Eberhardt, A.; Liang, K. S.; Eisenberger, P., Epitaxy and chainlength dependent strain in self-assembled monolayers. Journal of Chemical Physics 1997, 106, 1600-1608.

11. Poirier, G. E., Characterization of Organosulfur Molecular Monolayers on Au(111) using Scanning Tunneling Microscopy. Chemical Reviews 1997, 97 (4), 1117-1128.

12. Poirier, G. E.; Pylant, E. D., The Self-Assembly Mechanism of Alkanethiols on Au(111). Science 1996, 272 (5265), 1145-1148.

13. Allinger, N. L., Conformational analysis. 130. MM2. A hydrocarbon force field utilizing V1 and V2 torsional terms. Journal of the American Chemical Society 1977, 99 (25), 8127-8134. 